
\title{
Two-dimensional Stiefel-Whitney insulators in liganded Xenes
}

\author{
Mingxiang Pan $\mathbb{D}^{1}$, Dexin $\mathrm{Li}^{1}$, Jiahao Fan ${ }^{1}$ and Huaqing Huang $\mathbb{D}^{1,2,3 凶}$
}

Two-dimensional (2D) Stiefel-Whitney insulator (SWI), which is characterized by the second Stiefel-Whitney class, is a class of topological phases with zero Berry curvature. As an intriguing topological state, it has been well studied in theory but seldom realized in realistic materials. Here we propose that a large class of liganded Xenes, i.e., hydrogenated and halogenated 2D group-IV honeycomb lattices, are 2D SWIs. The nontrivial topology of liganded Xenes is identified by the bulk topological invariant and the existence of protected corner states. Moreover, the large and tunable bandgap (up to $3.5 \mathrm{eV}$ ) of liganded Xenes will facilitate the experimental characterization of the 2D SWI phase. Our findings not only provide abundant realistic material candidates that are experimentally feasible but also draw more fundamental research interest towards the topological physics associated with StiefelWhitney class in the absence of Berry curvature.

npj Computational Materials (2022)8:1 ; https://doi.org/10.1038/s41524-021-00695-2

\section{INTRODUCTION}

With the rapid progress of topological states, the concept of Berry curvature and associated topological invariants, such as Chern numbers ${ }^{1,2}$, mirror or spin Chern numbers ${ }^{3-7}$, and Fu-Kane invariants ${ }^{8,9}$, have been widely applied to condensed matter physics. Recently, a class of topological state with zero Berry curvature, which is characterized by the Stiefel-Whitney (SW) class, was proposed in spinless systems with space-time inversion symmetry $I_{S T}=P T$ or $C_{2 z} T$, where $P, T$, and $C_{2 z}$ are spatial inversion, time-reversal, and two-fold rotation symmetry, respectively ${ }^{10-15}$. This is the so-called SW insulator (SWI), which is topologically distinguished by a different topological invariant, i.e., the second SW number $w_{2}{ }^{16}$. Different from topological states associated with Chern class which possess topological boundary states due to the bulk-boundary correspondence, a 2D SWI features topologically protected corner states in the presence of additional chiral symmetry, indicating it is also a special class of $2 \mathrm{D}$ second-order topological insulators $\left(\right.$ SOTIs) ${ }^{17-21}$. So far, SOTIs have been proposed in various systems, including crystalline solids and artificial structures ${ }^{22-31}$. In the pioneering work of Lee et al. $^{32}$, the bulk topology of monolayer graphdiyne, which is a candidate for 2D SOTI, was characterized by a nontrivial second SW number $w_{2}=1$, indicating that it is also a candidate material for 2D SWI. However, the recently proposed 2D SWI was mainly studied in theory but seldom in realistic materials, which greatly hinders the experimental study of SWIs. It is thus emergent to search for 2D SWIs in realistic materials.

Meanwhile, in the field of 2D materials, a monoelemental class of $2 \mathrm{D}$ honeycomb crystals termed Xenes (X refers to $\mathrm{C}, \mathrm{Si}, \mathrm{Ge}, \mathrm{Sn}$, and so on $)^{33-35}$ have attracted tremendous attention as they provide an ideal platform to explore various topological physics. More than a dozen different topological phases, including the quantum spin Hall $(\mathrm{QSH})^{36-39}$, quantum anomalous $\mathrm{Hall}^{40}$, quantum valley Hall states ${ }^{41-43}$, and topological superconductors ${ }^{44-47}$, are predicted to emerge in Xenes, and these topological states are easily tuned, for example, by chemical functionalization with ligands. In particular, depending on the type of ligands, hydrogenated, or halogenated derivatives of Xene can be largegap QSH or trivial insulators with tunable gaps ${ }^{36,37}$.
In this work, we extend the theoretical prediction and experimental applicability of the topological physics associated with SW class by recognizing that the liganded Xene family $\mathrm{XL}$ $(\mathrm{X}=\mathrm{C}, \mathrm{Si}, \mathrm{Ge}, \mathrm{Sn}, \mathrm{L}=\mathrm{H}, \mathrm{F}, \mathrm{Cl}, \mathrm{Br}, \mathrm{I})$, a large, well studied, and readily synthesizable class of materials ${ }^{48-51}$, are 2D SWIs. Based on firstprinciples calculation and theoretical analysis, the chemical bonding configuration, bulk topological invariant, and in-gap topological corner states are calculated to identify the SW topology. Moreover, the large and tunable bandgaps of liganded Xenes will largely facilitate experimentally observing in-gap corner states. Since some liganded Xenes have been experimentally synthesized, we believe our proposal has strong feasibility to be detected by transport and STM measurements, which may draw immediate experimental attention.

\section{RESULTS \\ SW topology in hydrogenated graphene}

We begin our discussion by introducing the atomic structure and associated crystalline symmetry. Since the compounds in the ligand-terminated Xenes family have similar crystal structures and electronic structures, we take the single-side and double-side hydrogenated graphene (also named graphane), denoted as $s-\mathrm{CH}$ and $d-\mathrm{CH}$, as an example hereafter. Figure $1 \mathrm{a}$ and $\mathrm{b}$ shows crystal structures for $d-\mathrm{CH}$ and $s-\mathrm{CH}$, respectively. The $d-\mathrm{CH}$ with $\mathrm{H}$ alternating on both sides of the nanosheet is in a buckled hexagonal honeycomb structure with space group $164\left(D_{3 d}^{3}\right)$ including inversion symmetry $P$, while the $s-C H$ is in a planar configuration with space group $183\left(C_{6 v}^{1}\right)$, which contains $C_{2 z}$, the two-fold rotation symmetry about the $z$-axis. As time-reversal symmetry $T$ exists in both systems, therefore, the space-time inversion symmetry required for $2 \mathrm{D}$ SWIs are $I_{S T}=P T$ and $C_{2 z} T$ for $d-\mathrm{CH}$ and $\mathrm{s}-\mathrm{CH}$, respectively. The optimal lattice constants are 2.54 and $2.84 \AA$ for $d-\mathrm{CH}$ and $s-\mathrm{CH}$, respectively, which are consistent with previous reports ${ }^{52,53}$. We further confirm the stability of $d$ and $s$ - $\mathrm{CH}$ by phonon calculations that show no imaginary frequency (Supplementary Fig. 4).

For the hydrogenated graphene structures, $\mathrm{H}$ atoms directly couple to the half-filled $p_{z}$ orbitals in intrinsic graphene, thereby

${ }^{1}$ School of Physics, Peking University, 100871 Beijing, China. ${ }^{2}$ Collaborative Innovation Center of Quantum Matter, 100871 Beijing, China. ${ }^{3}$ Center for High Energy Physics, Peking University, 100871 Beijing, China. ${ }^{\circledR}$ email: huaqing.huang@pku.edu.cn 

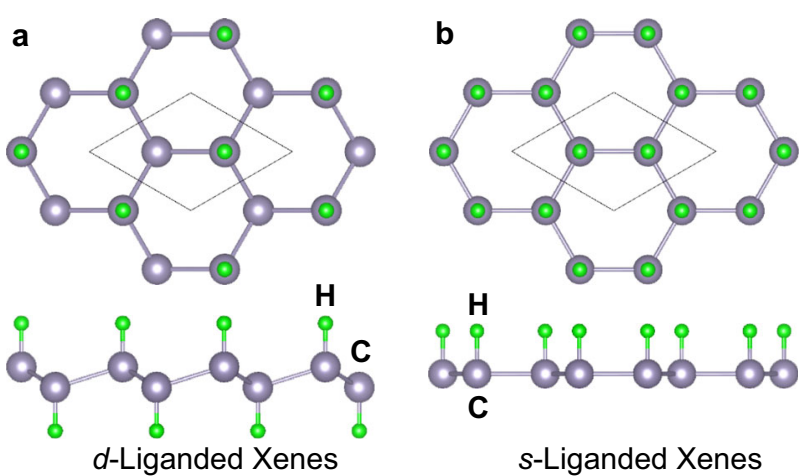

$s$-Liganded Xenes

Fig. 1 Crystal structure of liganed Xene. a, b Crystal structure for double-side and single-side liganded Xenes (e.g., $d-\mathrm{CH}$ and $s-\mathrm{CH}$ ) from the top (side) view [upper (lower)]. The gray and green spheres represent $\mathrm{C}$ and $\mathrm{H}$ atoms, respectively.

removing $\pi$ bonding and forming $\mathrm{H}-\mathrm{C} \sigma$ covalent states (Supplementary Fig. 3). The structural buckling in $d-\mathrm{CH}$ further enhances a $s p^{3}$ hybridization of $C$ atoms. There are in total five covalent bonds within the unit cell, including three $\mathrm{C}-\mathrm{C}$ and two $\mathrm{H}-\mathrm{C}$ bonds. Since the valence electron configuration of $\mathrm{C}$ and $\mathrm{H}$ are $2 s^{2} 2 p^{2}$ and $1 s^{1}$, covalent bonding states are fully occupied with two electrons per bonding state. Therefore, the system tends to be an insulator with the Fermi level lying in the gap between bonding and antibonding states. As shown in Fig. $2 a$ and $d, d-C H$ $(s-\mathrm{CH})$ is indeed an insulator with a direct (indirect) gap. Interestingly, the bandgap of $s-\mathrm{CH}(\sim 1.4 \mathrm{eV})$ is much smaller than that of $d-\mathrm{CH}(\sim 3.46 \mathrm{eV})$. Although the lack of structural corrugation in $s-\mathrm{CH}$ leads to deviations away from the $s p^{3}$ hybridization, singleside hydrogenation brings $\mathrm{H}$ atoms closer together, which naturally results in a much larger repulsion among the $\mathrm{H}-\mathrm{C} \sigma$ bonding states. As a consequence, the occupied band derived from $\mathrm{H}-\mathrm{C}$ bonds [marked by "-" at $\Gamma$ in Fig. $2 \mathrm{~d}$ ] shifts upwards in energy that gives rise to a smaller indirect bandgap of $s-\mathrm{CH}$.

To get a better understanding of the hybridization, covalency, and ionicity of chemical bonds in $d$ - and $\mathrm{s}-\mathrm{CH}$, we construct maximally localized Wannier functions (MLWFs) from the five occupied valence bands. As shown in Fig. $2 b$ and e, it is clear that these MLWFs display the character of $\sigma$-bonded combinations of mixed $s p^{2}-s p^{3}$ hybrids, providing an intuitive chemical signature of covalent bonds. Moreover, it can be seen that the MLWFs of $\mathrm{H}-\mathrm{C}$ covalent bonds and their Wannier charge centers are shifted towards $\mathrm{H}$ due to the difference of electronegativity between $\mathrm{H}$ and $\mathrm{C}$.

The common covalent $\sigma$ bonding states have the average charge center located around the middle of the bond, implying the unconventional feature of the mismatch between average electronic centers and atomic positions (also known as obstructed atomic limits $\left.{ }^{54,55}\right)$. This is a direct indicator of nontrivial band topology. We then perform a band representation (BR) analysis based on the theory of topological quantum chemistry ${ }^{56-59}$. The BR of trivial atomic insulators is solved to be a sum of atomicorbital-induced BRs (aBRs), while that of unconventional materials, such as SOTIs, must be a combination of some aBRs and an essential BR from an empty Wyckoff position where no atom exists $^{60,61}$. The BR decomposition of $d-\mathrm{CH}$ is $A_{1} @ 2 d+A_{g} @ 3 e$. As shown in Fig. $2 c$, the $C$ atoms are located at $2 d$ site of space group 164 , while $3 e$ sites of the essential BR are the $C-C$ bond centers, which correspond to $h_{3 c}^{(\overline{3})}$ primitive generator class of SOTIs in the Benalcazar et al.'s notation ${ }^{55}$. This implies that there is no net dipole in the plane and the corner charge fractionalization will be $\mathrm{e} / 2$ in each $\pi / 6$ sector. Similarly, we found the BR decomposition of $s-\mathrm{CH}$ is $A_{1} @ 2 b+A_{1} @ 3 c$, also indicating its nontrivial bulk topology (see Supplementary Table I and II).

Physically, the nontrivial bulk topology of $d$ - and $s-\mathrm{CH}$ can also be understood from the intuitive picture of double band inversion ${ }^{13,26,62-64}$. For $d-\mathrm{CH}$ with inversion symmetry $P$, we consider $N_{\text {occ }}^{-}(\Pi)$, the number of occupied bands with negative parity at time-reversal invariant momenta (TRIM, $\Pi=\Gamma$ and three $M$ points). It is found that $N_{\text {occ }}^{-}(M)-N_{\text {occ }}^{-}(\Gamma)=2$, as shown in Fig. 2a. This indicates a double band inversion, because the system cannot be adiabatically connected to the trivial atomic insulator limit where the parity representations at TRIM must be the same. Importantly, the second-order band topology of 2D inversionsymmetric spinless systems can be characterized by the second SW number $w_{2}{ }^{13,14,16,29,32}$, which is determined by

$$
(-1)^{W_{2}}=\prod_{\Pi \in \text { TRIM }}(-1)^{\left\lfloor N_{\text {occ }}^{-}(\Pi) / 2\right\rfloor},
$$

where $\lfloor\cdot\rfloor$ is the floor function. Therefore, the double band inversion gives rise to a nontrivial $w_{2}=1$, demonstrating that it belongs to the nontrivial SW class. Alternatively, $w_{2}$ for $s-\mathrm{CH}$ with $\mathrm{C}_{2 z} T$ symmetry can be obtained by tracing the Wilson loop spectra (see Fig. 3a). It is proved that $w_{2}$ is given by the parity of the number of spectral crossing at $\Theta=\pi$, where $\Theta$ indicates the phase eigenvalue of the Wilson loop operator ${ }^{13-15}$, as detailed in the "Methods" section and Supplementary Figs. 1 and 2. Due to a similar double band inversion with respect to $C_{2 z}$ in $s-C H$, as shown in Fig. $2 b$, we found a nontrivial $w_{2}=1$, confirming it is a SWI (see Fig. 3b).

To explicitly identify the second-order topology in $d$ - and $s-\mathrm{CH}$, we calculate the fractional corner charge $Q_{\text {corner, }}$ which is a bulk topological index for classifying SOTIs ${ }^{55}$. In $2 \mathrm{D}$ insulators with $C_{6}$ and $T$ symmetries (e.g., $s-\mathrm{CH}$ ), it can be evaluated $\mathrm{as}^{55}$

$Q_{\text {corner }}=\frac{\mathrm{e}}{4}\left[M_{1}^{(2)}\right]+\frac{\mathrm{e}}{6}\left[K_{1}^{(3)}\right] \bmod \mathrm{e}$,

where $\left[\Pi_{p}^{(n)}\right]=\# \Pi_{p}^{(n)}-\# \Gamma_{p}^{(n)}$ and $\# \Pi_{p}^{(n)}$ are defined as the number of bands below the bandgap with $C_{n}$ rotation eigenvalues $\Pi_{p}=\exp \left[\frac{2 \pi i(p-1)}{n}\right]$ for $p=1,2, \cdots, n$. $\Pi$ stands for high symmetric point $M$ and $K$. For $d-C H$ with $S_{6}$ symmetry, the above formula should be modified by replacing $\left[M_{1}^{(2)}\right]$ with $\left[M_{ \pm}^{(i)}\right]$, which is the difference in the number of bands with inversion eigenvalue even/odd between $M$ and $\Gamma^{65}$. Based on the first-principles calculations, we have $\left[M_{1}^{(2)}\right]=-2,\left[K_{1}^{(3)}\right]=0$ for $s-C H$ and $\left[M_{ \pm}^{(i)}\right]= \pm 2,\left[K_{1}^{(3)}\right]=0$ for $d-C H$. Therefore, $Q_{\text {corner }}=\mathrm{e} / 2$, indicating both $s-\mathrm{CH}$ and $d-\mathrm{CH}$ to be $2 \mathrm{D}$ SOTIs ${ }^{66}$.

To further reveal their topological nature, the first-principles calculations are performed to directly verify the gapped topological edge states and in-gap topological corner states in $d$ - and $s$ $\mathrm{CH}$, which can be used as a fingerprint to distinguish them from other topological phases. As shown in Fig. 4a and d, an edge band appears throughout the gap and detaches from bulk states of a zigzag nanoribbon. Since the first SW number $w_{1}$, which is equivalent to the Zak phase, is turned out to be trivial $\left(w_{1}=0\right)$, the existence of the 1D edge state is not due to the bulk polarization, but it is more like a dangling-bond state. In fact, different from the topologically protected helical edge states of 2D topological insulators, these edge states are less robust and can be removed by saturating the dangling bonds at edges with hydrogen atoms (see Supplementary Fig. 5). Similar flat edge states also occur in the monolayer $\beta$-Sb, which has been proved to be a 2D SOTI65,66.

Due to the modified bulk-boundary correspondence, the most direct indicator of SOTIs is the corner-localized in-gap states. To identify the corner topology of $d$ - and $s-\mathrm{CH}$, we calculate the energy spectrum for hexagonal nanodisks with $\sim 500$ atoms. In order to remove dangling-bond edge states from the bulk gap, we passivate the edges with hydrogen atoms (Supplementary Fig. 6). The energy spectrum for the nanodisk of $d-\mathrm{CH}$ and $s-\mathrm{CH}$ are plotted in Fig. $4 \mathrm{~b}$ and $\mathrm{e}$, respectively. Remarkably, there are six states that are degenerate at the Fermi level inside the bulk gap. Moreover, an 

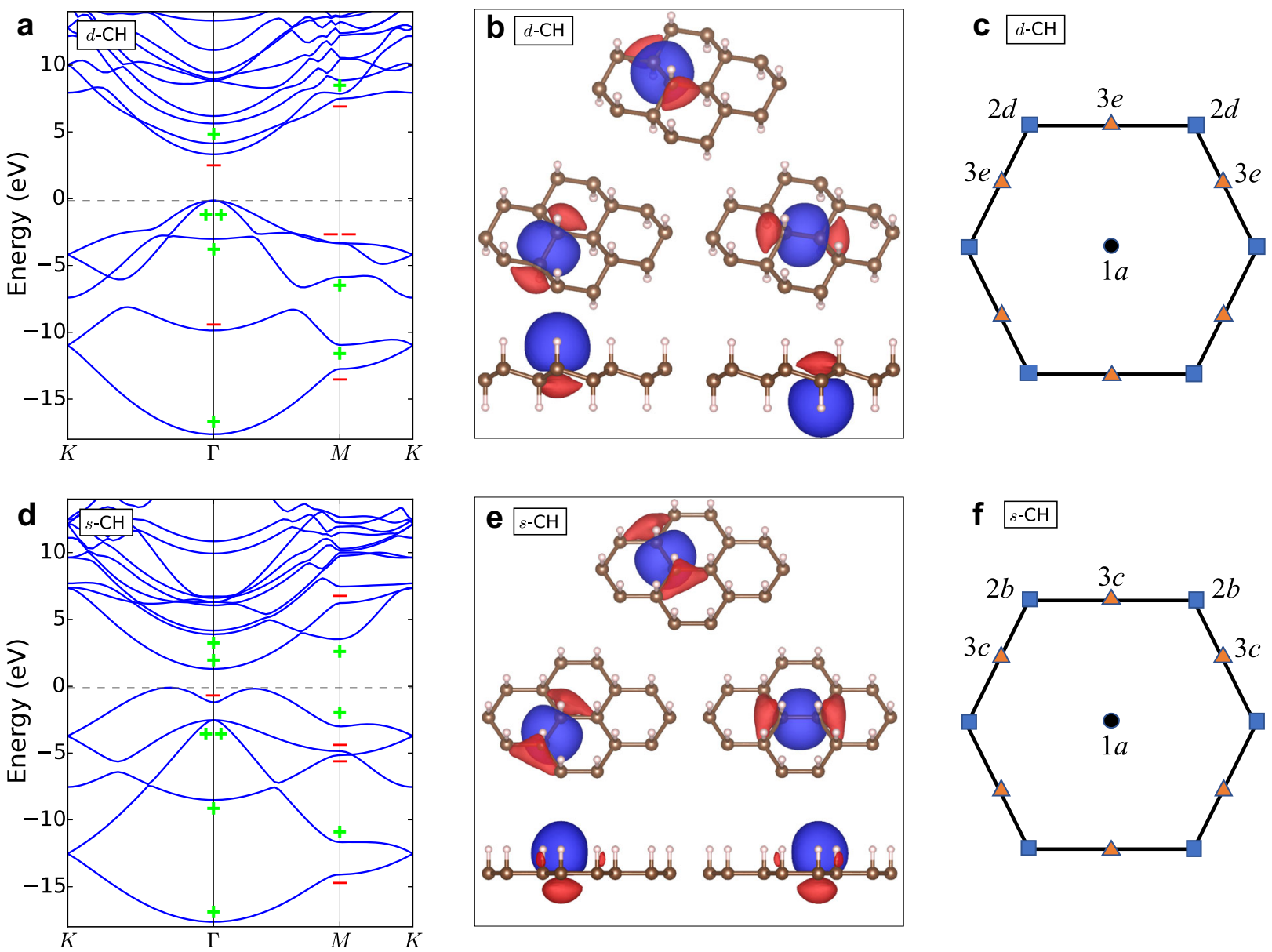

Fig. 2 Electronic structure analysis of hydrogenated graphene. Band structure of a $d-\mathrm{CH}$ and $\mathbf{d} s-\mathrm{CH}$. \pm marks Bloch states having opposite parities with respect to inversion or $C_{2 z}$ operation at high-symmetry points of the Brillouin zone. Isosurface contours of maximally localized Wannier functions (MLWFs) constructed from the five valence bands of $\mathbf{b} d-\mathrm{CH}$ and $\mathbf{e} s-\mathrm{CH}$, displaying the character of $\sigma$-bonded combinations of $s p^{3}$ hybrids (blue for positive value and red for negative). Wyckoff positions for $\mathbf{c} d$-CH with space group 164 and $\mathbf{f} s$ - $\mathrm{CH}$ with space group 183. The main Wyckoff sites are indicated by a black dot, blue squares ( $\mathrm{C}$ atoms) and orange triangles (empty sites), respectively.
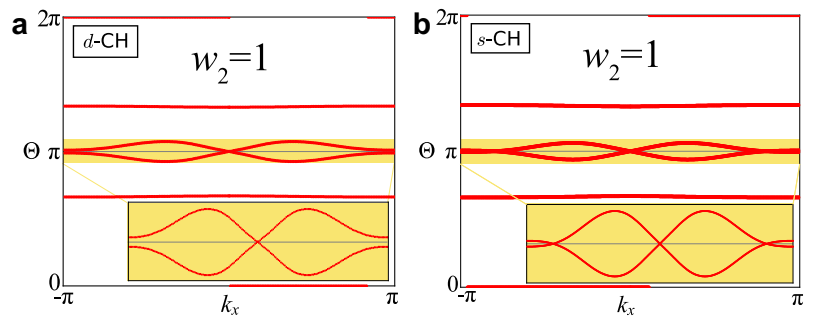

Fig. 3 The Wilson loop spectrum. The number of crossing points on $\Theta=\pi$ is 1 for $\mathbf{a} d-\mathrm{CH}$ and 3 for $\mathbf{b} s-\mathrm{CH}$, indicating a nontrivial second SW number $w_{2}=1$ for both systems. The insets show zoomed-in plots of the spectrum around $\Theta=\pi$.

emergent chiral (sublattice) symmetry, which derives from the bipartite honeycomb lattice, pins the corner modes in the mid of the gap. As shown in Fig. $4 \mathrm{c}$ and $\mathrm{f}$, these states are well localized at six corners of the hexagonal sample, confirming that they are the topological corner states. At exact half-filling, three of the six states are occupied, leading to a fraction corner charge of $Q_{\text {corner }}=e / 2$ per corner, which is consistent with the above analysis.

\section{Other liganded Xenes}

In addition, we also investigated other liganded Xenes. They are counterparts of hydrogenated graphene, corresponding to the silicene, germanene, and stanene monolayer saturated by hydrogen or halogen. Some of these materials, such as hydrogenated graphene ${ }^{67-69}$ and germanene ${ }^{70}$, or fluorinated graphene (also named fluorographene), have been experimentally synthesized ${ }^{70-73}$. Their topological properties have been carefully investigated according to the conventional classification of timereversal $Z_{2}$ topology. Owing to their negligible spin-orbit coupling, some liganded Xenes are identified as topologically trivial. However, this argument does not forbid an SW topology with zero Berry curvature.

Based on systematic calculations, we found that those prior trivial liganded Xenes are SWIs actually (see Table 1 and Supplementary Figs. 7-10). Since the $\pi$ orbitals are saturated by $\mathrm{H}$ or $\mathrm{F}$, these compounds become insulators with bandgaps in a wide range. Remarkably, the bandgaps of single-side hydrogenated germanane $(s-\mathrm{GeH})$ and stanene $(s-\mathrm{SnH})$ are larger than that of double-side hydrogenated ones, which is different from that of hydrogenated graphene. This is because the $\mathrm{H}-\mathrm{X} \sigma$ level does not shift to the valence band maximum due to the weaker repulsion between these states at large distances. Furthermore, among halogenated Xenes, more electronegative ligands (e.g., from $\mathrm{F}$ to I in halogens) tend to withdraw electron density from the Xene framework, and lower the energy of the ligand-X s-orbital antibonding levels at the conduction band minimum ${ }^{33,74}$. Therefore, the trend of bandgap reduction and the topological phase 

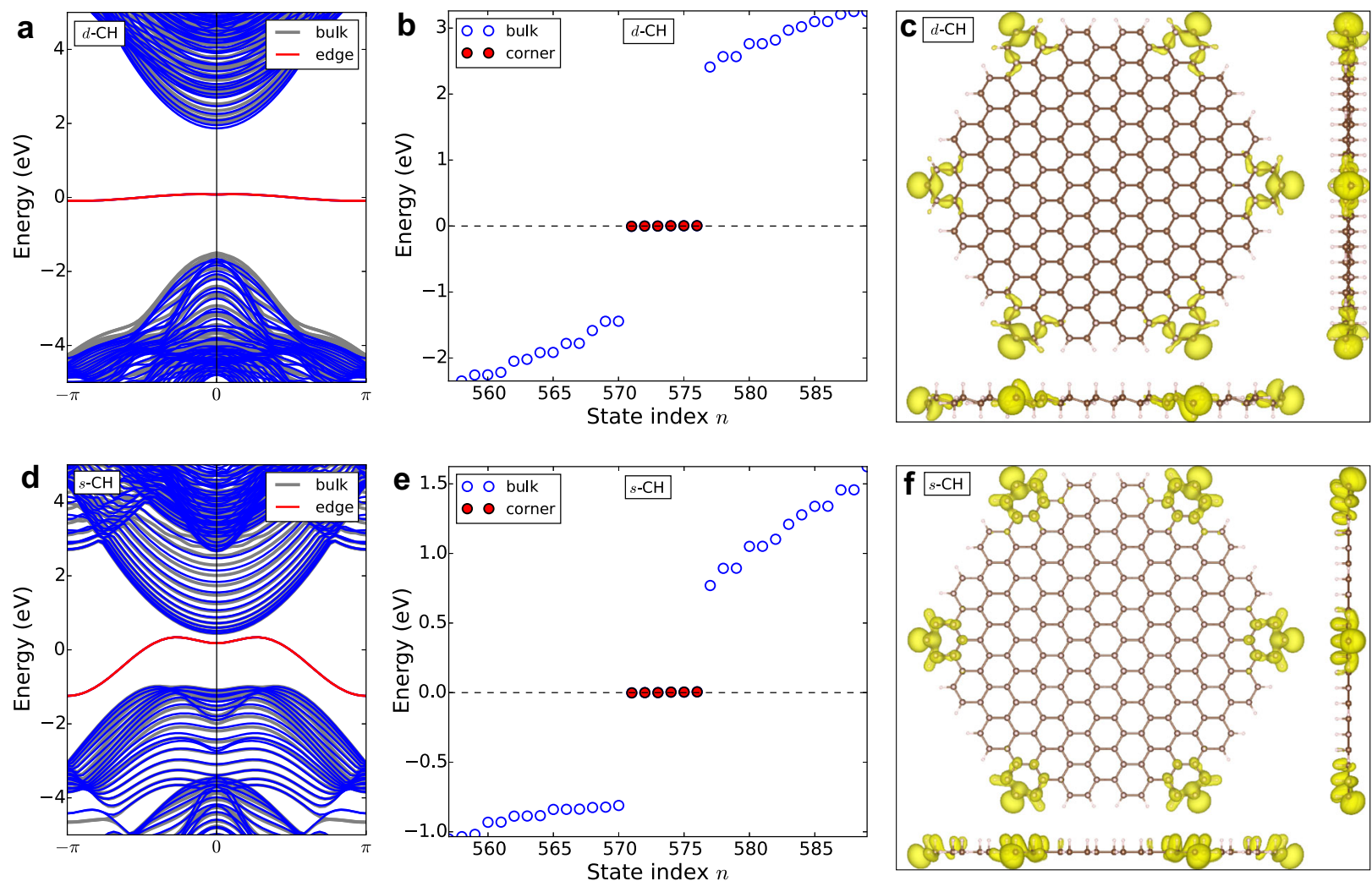

Fig. 4 SWI in $\mathbf{d}-\mathrm{CH}$ and s-CH honeycomb monolayer. a-c correspond to results for $d-\mathrm{CH}$ and $\mathbf{d}-\mathbf{f}$ correspond to results for $s-\mathrm{CH}$. a, $\mathbf{d}$ Band structure of a nanoribbon of hydrogenated graphene with (gray) and without (blue and red) hydrogen saturation. The flat edge bands are marked in red. $\mathbf{b}$, e The energy spectrum of a hexagonal-shaped nanodisk with H-saturated edges. $\mathbf{c}$, $\mathbf{f}$ Top and side view of the real-space charge distribution of corner states around the Fermi level.

Table 1. Topological phases of single-side (s-) or double-side $(d-)$ liganded Xene.

\begin{tabular}{|c|c|c|c|c|c|c|}
\hline & $w_{2} / Z_{2}$ & $\begin{array}{l}s- \\
a(\AA)\end{array}$ & $E_{g}(\mathrm{eV})$ & $w_{2} / Z_{2}$ & $\begin{array}{l}d- \\
a(\AA)\end{array}$ & $E_{g}(\mathrm{eV})$ \\
\hline $\mathrm{CH}$ & SWI & 2.84 & $1.40^{53}$ & SWI & 2.54 & $3.47^{52,68}$ \\
\hline $\mathrm{CF}$ & SWI & $3.51^{*}$ & 0.70 & SWI & 2.60 & $3.09^{72}$ \\
\hline $\mathrm{SiH}$ & SWI & 4.15 & 1.86 & SWI & 3.89 & 2.19 \\
\hline SiF & SWI & 4.32 & 1.30 & SWI & 3.95 & 0.68 \\
\hline $\mathrm{SiCl}$ & QSH & $5.00^{*}$ & 0.02 & SWI & 3.94 & 1.28 \\
\hline $\mathrm{SiBr}$ & QSH & $5.45^{*}$ & 0.05 & SWI & 3.97 & 1.20 \\
\hline Sil & QSH & $6.15^{*}$ & 0.09 & SWI & 4.06 & 0.55 \\
\hline $\mathrm{GeH}$ & SWI & 4.36 & 1.57 & SWI & 4.08 & $0.98^{70}$ \\
\hline GeF & QSH & 4.64 & 0.11 & SWI & 4.22 & $0.17^{35,37}$ \\
\hline $\mathrm{GeCl}$ & QSH & $5.29^{*}$ & 0.12 & SWI & 4.24 & $0.37^{35,37}$ \\
\hline $\mathrm{GeBr}$ & QSH & $5.67^{*}$ & 0.14 & SWI & 4.25 & $0.06^{35,37}$ \\
\hline Gel & QSH & $6.28^{*}$ & 0.17 & QSH & 4.32 & $0.30^{35,37}$ \\
\hline $\mathrm{SnH}$ & SWI & 4.99 & 1.32 & SWI & 4.71 & 0.47 \\
\hline $\mathrm{SnF}$ & QSH & 5.24 & 0.24 & QSH & 5.01 & $0.29^{36}$ \\
\hline $\mathrm{SnCl}$ & QSH & 5.58 & 0.24 & QSH & 4.93 & $0.26^{36}$ \\
\hline $\mathrm{SnBr}$ & QSH & $5.84^{*}$ & 0.25 & QSH & 4.91 & $0.29^{36}$ \\
\hline Snl & metal & $6.06^{*}$ & 0 & QSH & 4.90 & $0.34^{36}$ \\
\hline
\end{tabular}

The bandgaps $\left(E_{g}\right)$ are obtained from first-principles calculations or refer to experiments. $W_{2} / Z_{2}$ denotes the type of topological states (i.e., the $S W I$ or QSH phase). Star $\left(^{*}\right)$ mark energetically unfavorable $s$-liganded structures, which have much larger (>15\%) lattice constants $a$ than its $d$-counterpart. transition from SOTI to QSH are observed from $\mathrm{F}$ to I in both single and double-side halogenated Xenes.

Finally, for experimental detection on 2D SWIs, it is preferred to have the corner states sitting deep in the bulk gap. First, the bandgaps of liganded Xenes are large and tunable by the saturation position (s- or $d-$ ), the ligand type (hydrogen or halogens), and external perturbations such as electric field and strain. Second, the emergent approximate chiral (sublattice) symmetry of the honeycomb lattice structure pins the corner states approximately in the middle of the gap. All these features will facilitate the experimental characterization of the SWI phase in liganded Xenes. Moreover, given that some candidate materials have already been synthesized successfully in experiments ${ }^{48-51}$, the SWI phase is highly accessible, and may already be realized in existing materials.

\section{DISCUSSION}

In summary, we have demonstrated the 2D SWI phase in a large class of hydrogenated and halogenated Xenes that are experimentally feasible. The nontrivial topological nature of these materials is identified through the nontrivial second SW number $w_{2}=1$ and the existence of in-gap topological corner states. The candidate materials with bandgaps as large as $3.5 \mathrm{eV}$ will facilitate experimentally detecting in-gap corner states by STM measurements. Our results enrich the topological physics associated with SW class, and greatly extend the territory of candidate materials for 2D SWIs. In addition, it is also possible to realize 3D weak and strong SWIs by stacking these 2D SWI candidate materials. These discoveries may draw more fundamental research interests of Xenes, and provide a practical avenue for the realization of SWIs in real materials that are experimentally feasible. 


\section{METHODS}

\section{First-principles calculations}

We perform the first-principles calculations within the framework of density functional theory using the Vienna ab initio simulation package ${ }^{75}$. The exchange-correlation functional is treated using the Perdew-Burke-Ernzerhof (PBE) generalized-gradient approximation ${ }^{76}$. The predicted topology is further verified by using the Heyd-Scuseria-Ernzerhof (HSE) hybrid functional ${ }^{77}$. The negligible spin-orbit coupling is ignored in our calculations. We also generate MLWFs for the analysis of chemical bonding ${ }^{78,79}$.

\section{Wilson loop method}

The first SW number $w_{1}$ and second SW number $w_{2}$ can be calculated using the Wilson loop. The Wilson loop is defined as a path ordered product of the exponential of Berry connections,

$W_{\left(k_{1}+2 \pi, k_{2}\right) \leftarrow\left(k_{1}, k_{2}\right)}=\lim _{N \rightarrow \infty} F_{N-1} F_{N-2} \cdots F_{1} F_{0}$,

where $\left(k_{1}, k_{2}\right)$ parametrizes the $2 \mathrm{D}$ Brillouin zone, and $F_{i}$ is the overlap matrix whose elements of $F_{i}$ are given by

$\left[F_{i}\right]_{m n}=\left\langle u_{m}\left(2 \pi(i+1) / N, k_{2}\right) \mid u_{n}\left(2 \pi i / N, k_{2}\right)\right\rangle$.

The spectrum of Wilson loop is gauge invariant. The topological property of the system is determined by the phase factors $\theta_{m}\left(k_{2}\right) \in(-\pi, \pi]$ of the eigenvalues $\lambda_{m}\left(k_{2}\right)$ of Wilson operator, i.e., $\theta_{m}\left(k_{2}\right)=\operatorname{Imlog} \lambda_{m}\left(k_{2}\right)$. Computed along the $k_{1}$ direction parallel to the reciprocal lattice vector $G_{1}$ from $\left(k_{1}, k_{2}\right)$, the set of Wilson loop eigenvalues $\left\{e^{i \theta_{m}\left(k_{2}\right)}\right\}$ indicates the position of Wannier centers at given $k_{2}$, and the corresponding total charge polarization is given by $p_{1}=\frac{1}{2 \pi} \sum_{m=1}^{N_{\text {occ }}} \theta_{m}\left(k_{2}\right)$, which is equivalent to the first SW number $w_{1}$ in $k_{1}$ direction. The Wilson loop spectrum of liganded Xenes in the SWI phase shows that $w_{1}=0$ in both $k_{1}$ and $k_{2}$, indicating that the total polarization is zero.

The second SW number $w_{2}$ on a torus of the 2D Brillouin zone indicates whether the Wilson loop operator $\Theta$ can be continuously deformed to the identity operator or not, modulo an even number of winding on noncontractible cycles. Accordingly, the parity of the number of crossing points on $\Theta=\pi$ gives the second SW number $w_{2}$. Specifically, a spectrum corresponds to $w_{2}=0\left(w_{2}=1\right)$ if the parity is even (odd).

\section{Note added to proof}

After submission, we become aware of an independent work on arXiv recently ${ }^{80}$, where the results of liganded Xenes are consistent with ours.

\section{DATA AVAILABILITY}

All data used in this study are available from the corresponding author upon reasonable request.

Received: 4 September 2021; Accepted: 22 December 2021; Published online: 10 January 2022

\section{REFERENCES}

1. Thouless, D. J., Kohmoto, M., Nightingale, M. P. \& den Nijs, M. Quantized hall conductance in a two-dimensional periodic potential. Phys. Rev. Lett. 49, 405-408 (1982).

2. Niu, Q., Thouless, D. J. \& Wu, Y.-S. Quantized hall conductance as a topological invariant. Phys. Rev. B 31, 3372-3377 (1985).

3. Teo, J. C. Y., Fu, L. \& Kane, C. L. Surface states and topological invariants in threedimensional topological insulators: application to $b_{1-x} \mathrm{sb}_{x}$. Phys. Rev. $B$ 78, 045426 (2008)

4. Hsieh, T. H. et al. Topological crystalline insulators in the snte material class. Nat. Commun. 3, 982 (2012).

5. Sheng, D. N., Weng, Z. Y., Sheng, L. \& Haldane, F. D. M. Quantum spin-hall effect and topologically invariant chern numbers. Phys. Rev. Lett. 97, 036808 (2006).

6. Fukui, T. \& Hatsugai, Y. Topological aspects of the quantum spin-hall effect in graphene: $z_{2}$ topological order and spin chern number. Phys. Rev. B 75, 121403 (2007).

7. Prodan, E. Robustness of the spin-chern number. Phys. Rev. B 80, 125327 (2009).

8. Fu, L. \& Kane, C. L. Time reversal polarization and a $Z_{2}$ adiabatic spin pump. Phys. Rev. B 74, 195312 (2006).
9. Fukui, T. \& Hatsugai, Y. Quantum spin hall effect in three dimensional materials: lattice computation of $z 2$ topological invariants and its application to bi and sb. J. Phys. Soc. Jpn. 76, 053702 (2007).

10. Zhao, Y. X., Schnyder, A. P. \& Wang, Z. D. Unified theory of $p t$ and $c p$ invariant topological metals and nodal superconductors. Phys. Rev. Lett. 116, 156402 (2016).

11. Zhao, Y. X. \& Lu, Y. pt-symmetric real dirac fermions and semimetals. Phys. Rev. Lett. 118, 056401 (2017).

12. Wang, K., Dai, J.-X., Shao, L. B., Yang, S. A. \& Zhao, Y. X. Boundary criticality of PTinvariant topology and second-order nodal-line semimetals. Phys. Rev. Lett. 125, 126403 (2020).

13. Ahn, J., Kim, D., Kim, Y. \& Yang, B.-J. Band topology and linking structure of nodal line semimetals with $Z_{2}$ monopole charges. Phys. Rev. Lett. 121, 106403 (2018).

14. Ahn, J., Park, S., Kim, D., Kim, Y. \& Yang, B.-J. Stiefel-whitney classes and topological phases in band theory. Chin. Phys. B 28, 117101 (2019).

15. Ahn, J., Park, S. \& Yang, B.-J. Failure of nielsen-ninomiya theorem and fragile topology in two-dimensional systems with space-time inversion symmetry: application to twisted bilayer graphene at magic angle. Phys. Rev. X 9, 021013 (2019).

16. Ahn, J. \& Yang, B.-J. Symmetry representation approach to topological invariants in $C_{2 z} t$-symmetric systems. Phys. Rev. B 99, 235125 (2019).

17. Benalcazar, W. A., Bernevig, B. A. \& Hughes, T. L. Quantized electric multipole insulators. Science 357, 61-66 (2017).

18. Schindler, F. et al. Higher-order topological insulators. Sci. Adv. 4, eaat0346 (2018).

19. Ezawa, M. Higher-order topological insulators and semimetals on the breathing kagome and pyrochlore lattices. Phys. Rev. Lett. 120, 026801 (2018).

20. Langbehn, J., Peng, Y., Trifunovic, L., von Oppen, F. \& Brouwer, P. W. Reflectionsymmetric second-order topological insulators and superconductors. Phys. Rev. Lett. 119, 246401 (2017).

21. Song, Z., Fang, Z. \& Fang, C. ( $d-2)$-dimensional edge states of rotation symmetry protected topological states. Phys. Rev. Lett. 119, 246402 (2017).

22. Schindler, F. et al. Higher-order topology in bismuth. Nat. Phys. 14, 918 (2018).

23. Yue, C. et al. Symmetry-enforced chiral hinge states and surface quantum anomalous hall effect in the magnetic axion insulator $\mathrm{bi}_{2-x} \mathrm{sm}_{x} \mathrm{se}_{3}$. Nat. Phys. 15 577-581 (2019).

24. Xu, Y., Song, Z., Wang, Z., Weng, H. \& Dai, X. Higher-order topology of the axion insulator euin as $_{2}$. Phys. Rev. Lett. 122, 256402 (2019).

25. Zhang, R.-X., Wu, F. \& Das Sarma, S. Möbius insulator and higher-order topology in $\mathrm{mnbi}_{2 n} \mathrm{te} \mathrm{3n+1}_{3 n}$. Phys. Rev. Lett. 124, 136407 (2020).

26. Wang, Z., Wieder, B. J., Li, J., Yan, B. \& Bernevig, B. A. Higher-order topology, monopole nodal lines, and the origin of large fermi arcs in transition metal dichalcogenides $x \mathrm{te}_{2}(x=$ Mo, W). Phys. Rev. Lett. 123, 186401 (2019).

27. Sheng, X.-L. et al. Two-dimensional second-order topological insulator in graphdiyne. Phys. Rev. Lett. 123, 256402 (2019).

28. Liu, B., Zhao, G., Liu, Z. \& Wang, Z. Two-dimensional quadrupole topological insulator in $\gamma$-graphyne. Nano Lett. 19, 6492-6497 (2019).

29. Park, M. J., Kim, Y., Cho, G. Y. \& Lee, S. Higher-order topological insulator in twisted bilayer graphene. Phys. Rev. Lett. 123, 216803 (2019).

30. Liu, B. et al. Higher-order band topology in twisted moiré superlattice. Phys. Rev. Lett. 126, 066401 (2021).

31. Chen, C. et al. Universal approach to magnetic second-order topological insulator. Phys. Rev. Lett. 125, 056402 (2020).

32. Lee, E., Kim, R., Ahn, J. \& Yang, B.-J. Two-dimensional higher-order topology in monolayer graphdiyne. npj Quantum Mater. 5, 1-7 (2020).

33. Molle, A. et al. Buckled two-dimensional xene sheets. Nat. Mater. 16, 163-169 (2017).

34. Zhao, A. \& Wang, B. Two-dimensional graphene-like xenes as potential topological materials. APL Mater. 8, 030701 (2020).

35. Bechstedt, F., Gori, P. \& Pulci, O. Beyond graphene: clean, hydrogenated and halogenated silicene, germanene, stanene, and plumbene. Prog. Surf. Sci. 96 100615 (2021).

36. $\mathrm{Xu}, \mathrm{Y}$. et al. Large-gap quantum spin hall insulators in tin films. Phys. Rev. Lett. 111, 136804 (2013).

37. $\mathrm{Si}, \mathrm{C}$. et al. Functionalized germanene as a prototype of large-gap two-dimensional topological insulators. Phys. Rev. B 89, 115429 (2014).

38. Liu, C.-C., Feng, W. \& Yao, Y. Quantum spin hall effect in silicene and twodimensional germanium. Phys. Rev. Lett. 107, 076802 (2011).

39. Kane, C. L. \& Mele, E. J. Quantum spin hall effect in graphene. Phys. Rev. Lett. 95 226801 (2005)

40. Wu, S.-C., Shan, G. \& Yan, B. Prediction of near-room-temperature quantum anomalous hall effect on honeycomb materials. Phys. Rev. Lett. 113, 256401 (2014).

41. Ezawa, M. Valley-polarized metals and quantum anomalous hall effect in silicene. Phys. Rev. Lett. 109, 055502 (2012). 
42. Ezawa, M. Spin valleytronics in silicene: quantum spin hall-quantum anomalous hall insulators and single-valley semimetals. Phys. Rev. B 87, 155415 (2013).

43. Ezawa, M. Monolayer topological insulators: Silicene, germanene, and stanene. J. Phys. Soc. Jpn. 84, 121003 (2015).

44. Wang, J., Xu, Y. \& Zhang, S.-C. Two-dimensional time-reversal-invariant topological superconductivity in a doped quantum spin-hall insulator. Phys. Rev. B 90, 054503 (2014)

45. Wang, C. et al. Type-ii ising superconductivity in two-dimensional materials with spin-orbit coupling. Phys. Rev. Lett. 123, 126402 (2019).

46. Falson, J. et al. Type-ii ising pairing in few-layer stanene. Science 367, 1454-1457 (2020).

47. Liao, M. et al. Superconductivity in few-layer stanene. Nat. Phys. 14, 344-348 (2018).

48. Mannix, A. J., Kiraly, B., Hersam, M. C. \& Guisinger, N. P. Synthesis and chemistry of elemental 2d materials. Nat. Rev. Chem. 1, 1-14 (2017).

49. Grazianetti, C., Martella, C. \& Molle, A. The xenes generations: a taxonomy of epitaxial single-element $2 \mathrm{~d}$ materials. Phys. Status Solidi Rapid Res. Lett. 14, 1900439 (2020).

50. Antonatos, N., Ghodrati, H. \& Sofer, Z. Elements beyond graphene: current state and perspectives of elemental monolayer deposition by bottom-up approach. Appl. Mater. Today 18, 100502 (2020).

51. Zhang, L. et al. Recent advances in hybridization, doping, and functionalization of 2d xenes. Adv. Funct. Mater. 31, 2005471 (2021).

52. Sofo, J. O., Chaudhari, A. S. \& Barber, G. D. Graphane: A two-dimensional hydrocarbon. Phys. Rev. B 75, 153401 (2007).

53. Pujari, B. S., Gusarov, S., Brett, M. \& Kovalenko, A. Single-side-hydrogenated graphene: density functional theory predictions. Phys. Rev. B 84, 041402 (2011).

54. Cano, J. et al. Building blocks of topological quantum chemistry: elementary band representations. Phys. Rev. B 97, 035139 (2018).

55. Benalcazar, W. A., Li, T. \& Hughes, T. L. Quantization of fractional corner charge in $C_{n}$-symmetric higher-order topological crystalline insulators. Phys. Rev. B 99, 245151 (2019)

56. Bradlyn, B. et al. Topological quantum chemistry. Nature 547, 298 (2017).

57. Vergniory, M. et al. A complete catalogue of high-quality topological materials. Nature 566, 480 (2019).

58. Tang, F., Po, H. C., Vishwanath, A. \& Wan, X. Comprehensive search for topological materials using symmetry indicators. Nature 566, 486 (2019).

59. Zhang, T. et al. Catalogue of topological electronic materials. Nature 566, 475 (2019).

60. Gao, J. et al. Unconventional materials: the mismatch between electronic charge centers andatomic positions. Preprint at https://arxiv.org/abs/2106.08035 (2021).

61. Nie, S. et al. Application of topological quantum chemistry in electrides. Phys. Rev. B 103, 205133 (2021).

62. Hsu, C.-H. et al. Topology on a new facet of bismuth. Proc. Natl Acad. Sci. USA 116, 13255-13259 (2019)

63. Xiao, J. \& Yan, B. First-principles calculations for topological quantum materials. Nat. Rev. Phys. 3, 283-297 (2021).

64. Huang, H., Fan, J., Li, D. \& Liu, F. Generic orbital design of higher-order topological quasicrystalline insulators with odd five-fold rotation symmetry. Nano Lett. 21, 7056-7062 (2021).

65. Radha, S. K. \& Lambrecht, W. R. L. Buckled honeycomb antimony: higher order topological insulator and its relation to the kekulé lattice. Phys. Rev. B 102, 115104 (2020).

66. Schindler, F. et al. Fractional corner charges in spin-orbit coupled crystals. Phys. Rev. Res. 1, 033074 (2019).

67. Elias, D. C. et al. Control of graphene's properties by reversible hydrogenation: evidence for graphane. Science 323, 610-613 (2009).

68. Balog, R. et al. Bandgap opening in graphene induced by patterned hydrogen adsorption. Nat. Mater. 9, 315-319 (2010).

69. Whitener, K. E. Review article: Hydrogenated graphene: a user's guide. J. Vac. Sci. Technol. A 36, $05 \mathrm{G} 401$ (2018).

70. Bianco, E. et al. Stability and exfoliation of germanane: a germanium graphane analogue. ACS Nano 7, 4414-4421 (2013).

71. Jeon, K.-J. et al. Fluorographene: a wide bandgap semiconductor with ultraviolet luminescence. Acs Nano 5, 1042-1046 (2011).

72. Nair, R. R. et al. Fluorographene: a two-dimensional counterpart of teflon. Small 6, 2877-2884 (2010)
73. Zboril, R. et al. Graphene fluoride: a stable stoichiometric graphene derivative and its chemical conversion to graphene. Small 6, 2885-2891 (2010).

74. Van de Walle, C. G. \& Northrup, J. E. First-principles investigation of visible light emission from silicon-based materials. Phys. Rev. Lett. 70, 1116-1119 (1993).

75. Kresse, G. \& Furthmüller, J. Efficiency of ab-initio total energy calculations for metals and semiconductors using a plane-wave basis set. Comput. Mater. Sci. 6, 15 (1996).

76. Perdew, J. P., Burke, K. \& Ernzerhof, M. Pbe functional. Phys. Rev. Lett. 77, 3865 (1996).

77. Heyd, J., Scuseria, G. E. \& Ernzerhof, M. Hybrid functionals based on a screened coulomb potential. J. Chem. Phys. 118, 8207-8215 (2003).

78. Mostofi, A. A. et al. wannier90: A tool for obtaining maximally-localised wannier functions. Comput. Phys. Commun. 178, 685-699 (2008)

79. Marzari, N., Mostofi, A. A., Yates, J. R., Souza, I. \& Vanderbilt, D. Maximally localized wannier functions: Theory and applications. Rev. Mod. Phys. 84, 1419-1475 (2012).

80. Qian, S., Liu, C.-C. \& Yao, Y. Second order topological insulator state in hexagonal lattices and its abundant material candidates. Phys. Rev. B. 104, 245427 (2021).

\section{ACKNOWLEDGEMENTS}

We thank Jiaheng Gao and Zhijun Wang for the help in determining the BR decomposition. This work was supported by the National Natural Science Foundation of China (Grant No. 12074006), the National Key Research and Development Program of China (No. 2021YFA1401600), and the start-up fund from Peking University. The computational resources were supported by the high-performance computing platform of Peking University.

\section{AUTHOR CONTRIBUTIONS}

H.H. supervised the project. M.P. carried out the DFT calculations. H.H. wrote the manuscript. D.L. and J.F. commented on the manuscript. All the authors contributed to the manuscript.

\section{COMPETING INTERESTS}

The authors declare no competing interests

\section{ADDITIONAL INFORMATION}

Supplementary information The online version contains supplementary material available at https://doi.org/10.1038/s41524-021-00695-2.

Correspondence and requests for materials should be addressed to Huaqing Huang.

Reprints and permission information is available at http://www.nature.com/ reprints

Publisher's note Springer Nature remains neutral with regard to jurisdictional claims in published maps and institutional affiliations.

(i) Open Access This article is licensed under a Creative Commons Attribution 4.0 International License, which permits use, sharing, adaptation, distribution and reproduction in any medium or format, as long as you give appropriate credit to the original author(s) and the source, provide a link to the Creative Commons license, and indicate if changes were made. The images or other third party material in this article are included in the article's Creative Commons license, unless indicated otherwise in a credit line to the material. If material is not included in the article's Creative Commons license and your intended use is not permitted by statutory regulation or exceeds the permitted use, you will need to obtain permission directly from the copyright holder. To view a copy of this license, visit http://creativecommons. org/licenses/by/4.0/.

(c) The Author(s) 2022 